\title{
Exercise ameliorates the detrimental effect of chloroquine on skeletal muscles in mice via restoring autophagy flux
}

\author{
Dan JIANG, \#, Kai CHEN ${ }^{2, \#}$, Xuan $\mathrm{LU}^{1}$, Hong-jian GAO ${ }^{3}$, Zheng-hong $\mathrm{QIN}^{1}$, Fang $\mathrm{LIN}^{1, *}$ \\ ${ }^{1}$ Department of Pharmacology, Laboratory of Aging and Nervous Diseases (SZS0703), Soochow University School of Pharmaceutical \\ Science, Suzhou 215123, China; ${ }^{2}$ Department of Cancer, the First Affiliated Hospital of Soochow University, Suzhou 215006, China; \\ ${ }^{3}$ Electron Microscopy Core Laboratory, Shanghai Medical College, Fudan University, Shanghai 200032, China
}

Aim: To study the roles of autophagy in muscle establishment during long-term exercise in mice.

Methods: Female ICR mice were submitted to exercise training with a wheel running regimen: $6 \mathrm{~m} / \mathrm{min}, 15 \mathrm{~min} / \mathrm{time}, 3$ times/d (on 8:00, 14:00, and 20:00), $5 \mathrm{~d}$ /week for 2 months. The mice were treated with the autophagy activator trehalose (1\% aqueous solution as a daily drinking water) or the autophagy inhibitor chloroquine $(10 \mathrm{mg} / \mathrm{kg}$, ip, 5 times a week) before the training. Western blotting analysis, TUNEL staining, H\&E staining and transmission electron microscope were used to evaluate the activity of autophagy and the structure of the muscle fibers.

Results: The exercise training significantly stimulated the formation of autophagosomes, increased the LC3-II, cathepsin L and Bcl-2 levels, lowered the P62 level and increased the antioxidant capacity in the muscles. Meanwhile, the exercise training significantly improved the morphology of mitochondria, reduced the release of cytochrome $c$ from mitochondria to cytoplasm, and slightly decreased the apoptosis rate in the muscles. Administration of trehalose increased the level of autophagy and protected the muscle fibers from apoptosis. Administration of chloroquine blocked autophagy flux and exerted detrimental effects on the muscles, which were ameliorated by the exercise training.

Conclusion: Long-term regular exercise activates autophagy process associated with muscle establishment, and ameliorates the detrimental effects of chloroquine on skeletal muscles via restoring autophagy flux.

Keywords: autophagy; skeletal muscle; exercise; apoptosis; mitochondria; trehalose; chloroquine

Acta Pharmacologica Sinica (2014) 35: 135-142; doi: 10.1038/aps.2013.144; published online 16 Dec 2013

\section{Introduction}

It has long been recognized that regular endurance exercise exerts beneficial effects in a number of chronic diseases, including amyotrophy, osteoporosis, depression, and cardiovascular disease $^{[1]}$. In particular, the benefits of exercise can be observed in the skeletal muscles. Muscle contraction can metabolically and mechanically alter the muscle tissue and the organelles.

To maintain homeostasis, muscle cells require an efficient system for recycling proteins and eliminating protein aggregates and dysfunctional organelles. These actions are carried out through the process of macroautophagy ${ }^{[2,3]}$. During macroautophagy, double-membrane vesicles engulf regions

\footnotetext{
\# These authors contributed equally to this article.

* To whom correspondence should be addressed.

E-mail bluestonelin@hotmail.com

Received 2013-04-14 Accepted 2013-08-28
}

of the cytoplasm to clear substrate proteins and organelles ${ }^{[4-6]}$. However, the mechanisms and principles underlying the effects of exercise on muscle establishment are not completely understood. Masiero et al ${ }^{[7]}$ reported that muscle-specific deletion of a crucial autophagy gene, Atg7, caused accumulation of abnormal mitochondria, sarcoplasmic reticulum distension, disorganization of the sarcomere, and formation of aberrant concentric membranous structures, and it resulted in profound muscle atrophy and an age-dependent decrease in force. Thus, autophagy flux is important for preserving muscle mass and maintaining muscle fiber integrity.

Exercise plays an important role in the maintenance of muscle fitness in healthy muscle and under some diseases conditions $^{[8,9]}$. However, it is not very clear whether autophagy mediates the effects of exercise on muscle establishment. Ogura et $a l^{[10]}$ reported that a single session of exercise increased the LC3-II level. However, Kim et al ${ }^{[11]}$ reported that the expression of autophagosome-related proteins was attenu- 
ated by a single bout of moderate treadmill exercise in murine gastrocnemius skeletal muscle. Later, Jamart et al ${ }^{[12]}$ demonstrated the ultra-endurance exercise increased autophagy flux in human skeletal muscle. Wohlgemuth et al ${ }^{[13]}$ showed that a program of weight loss and exercise resulted in an increase in autophagy in older obese women. Using Bcl-2 knockout mice, He $e a^{[14]}$ demonstrated that $\mathrm{Bcl}-2$ was a crucial regulator of exercise-induced autophagy in vivo, and the induced autophagy contributed to the beneficial metabolic effects of exercise.

The quantity and quality of mitochondria in skeletal muscles are important for performance as well as for health. Sports training enables adaptive changes that increase the number of mitochondria and upregulate mitochondrial mass and function throughout the body to maintain metabolic homeostasis $^{[15]}$. The pathologies of muscle atrophy, diabetes, and aging are known to be associated with mitochondrial dysfunction $^{[16,17]}$. However, little research has focused on the role of autophagy in mitochondrial function in skeletal muscle after regular endurance exercise.

Excessive autophagy maybe also cause muscle atrophy ${ }^{[17,18]}$, so the "Goldilocks" principle must be applied to determine the level of autophagy activation that is "just right" for maintaining long-term muscle health ${ }^{[19]}$. We hypothesized that long-term regular exercise could activate autophagy to this "just right" level for muscle establishment and health maintenance. To test this hypothesis, we attempted to demonstrate the relationship between exercise and autophagy in vivo using a mouse aerobic exercise model. An autophagy inducer and inhibitor were used as well to confirm whether increased autophagy contributed to the enhancement of mitochondrial function and skeletal muscle mass.

\section{Materials and methods}

Animals

Female ICR mice were purchased from the Center for Experimental Animals of Soochow University (certificate № 20020008 , Grade II) at 7-8 weeks of age and were housed in standard rodent cages in a temperature- $\left(22 \pm 2.5^{\circ} \mathrm{C}\right)$, humidity (50\%-60\%), ventilation- and light-controlled (12 h:12 h lightdark cycle) environment with unrestricted access to water and food. The mice that were submitted to exercise training were trained with a wheel running regimen as follows: $6 \mathrm{~m} / \mathrm{min}$, $15 \mathrm{~min} /$ time, 3 times/d (8:00, 14:00, and 20:00, respectively), $5 \mathrm{~d} /$ week, for 8 weeks. To explore the role of autophagy, the autophagy inducer trehalose and the autophagy inhibitor chloroquine were selected ${ }^{[20-22]}$. The mice were treated with a $1 \%$ aqueous solution of trehalose (Sinozyme Biotechnology, Nanning, China) added to the daily drinking water or by intraperitoneal injection of chloroquine $(10 \mathrm{mg} / \mathrm{kg}$, Sigma, C6628-25G) 5 times a week.

\section{Transmission electron microscopic examination}

Samples of the rectus femoris muscle with dimensions of 1 $\mathrm{mm} \times 1 \mathrm{~mm} \times 3 \mathrm{~mm}$ were removed and fixed in $2.5 \%$ glutaraldehyde with phosphate buffer for $2 \mathrm{~h}$, then rinsed with 1 $\mathrm{mmol} / \mathrm{L}$ phosphoric acid solution and fixed in $1 \%$ osmium tetroxide for 2-3 h. The blocks were cut carefully into ultrathin $(0.07 \mu \mathrm{m})$ sections, which were stained with $3 \%$ uranyl acetate and lead citrate and then viewed under a JEOL JEM1400 electron microscope at East China University of Science and Technology (Shanghai, China).

\section{H\&E staining}

Samples of rectus femoris muscle were fixed in 10\% neutralbuffered formalin and cut into longitudinal sections of $5 \mu \mathrm{m}$. Then, the sections were dewaxed and rehydrated and were stained with hematoxylin (CAT Hematoxylin). The sections were observed under a light microscope at a magnification of $40 \times$.

\section{Western blotting analysis}

The whole lateralis muscle was homogenized in tissue lysing buffer. The primary antibodies used in this study were rabbit anti-LC3 (1:500, Abcam, ab62721), rabbit anti-p62/SQSTM1 (1:1000, Enzo Life Sciences), Bcl-2 (C-2) mouse monoclonal IgG1 (1:1000, Santa Cruz Biotechnology, 5640), rabbit antiBnip3 (1:500, Cell Signaling Technology, 3769), mouse antiGAPDH (1:1000, Advanced ImmunoChemical, 25007), mouse anti-histone H3 (1:1000, Cell Signaling Technology, 9715), and mouse anti-cytochrome c (1:200, Beyotime), rabbit anti-COX IV (1:1000, Beyotime). As secondary antibodies, horseradish peroxidase-conjugated anti-rabbit 711-035-152 and anti-mouse 715-035-150 (Jackson ImmunoResearch) were used. Immunoreactivity was detected with enhanced chemiluminescent autoradiography (Pierce, 34080) in accordance with the manufacturer's instructions. The levels of protein expression were quantitatively analyzed with Sigma Scan Pro 5.

\section{Terminal deoxynucleotidyl transferase dUTP nick-end labeling (TUNEL) assays}

Rectus femoris tissue was used to determine the apoptosis levels with the TUNEL assay (In Situ Cell Death Detection Kit, Roche Applied Science, 11684817910) following the manufacturer's instructions ${ }^{[23]}$.

\section{Determination of SOD, GSH, and MDA}

SOD, GSH and MDA in gastrocnemius muscle samples were determined using the Total Superoxide Dismutase Assay Kit with WST-1, the Glutathione Reductase Assay Kit, and the Lipid Peroxidation MDA Assay Kit (Beyotime Institute of Biotechnology, Haimen, China), respectively. The specific experimental procedures are available at http://www.beyotime. com.

\section{Extraction of mitochondria}

The mitochondria were extracted from the gastrocnemius tissue using a Tissue Mitochondria Isolation Kit. The specific experimental procedures are available at http://www.beyotime.com.

\section{Statistical analysis}

Statistical analysis was carried out by one-way ANOVA 
followed by Dunnett $t$-test or Student $t$-test (between two groups). The data are presented as the mean \pm standard deviation (SD). $P<0.05$ was considered statistically significant.

\section{Results}

\section{Exercise reduced chloroquine-induced muscle damage}

The mice were divided into six groups $(n=8)$ : untrained mice treated with saline (NS), untrained mice treated with trehalose (Tre), untrained mice treated with chloroquine $(\mathrm{Chl})$, trained mice (Exe), trained mice treated with trehalose (Exe+Tre), and trained mice treated with chloroquine (Exe+Chl). The body weight and food consumption of the mice were recorded regularly every week. Pretreatment with the autophagy inducer trehalose or the inhibitor chloroquine did not significantly change the body weight and was not closely associated with exercise (Figure 1A). The relative food consumption (g/10 g body weight) showed that the exercise training did not cause the mice to consume more food (Figure 1B). However, the relative abdominal fat weight ( $\mathrm{g} / \mathrm{g}$ body weight) of the exercisetrained mice was significantly lower than that of the non-exercise group. Similarly, exercise obviously reduced the weight of the intra-abdominal fat in the mice treated with trehalose or chloroquine (Figure 1C).

To characterize the internal structure of the muscle fibers, we observed the morphology of the rectus femoris stained with hematoxylin and eosin (H\&E). Under normal conditions, the myofilament was in a state of contraction, and the arrangement was orderly and neat. All nuclei were located at the edge of the muscle fibers, whereas muscle fibers in the group treated with the autophagy inhibitor chloroquine had been damaged, the structure became loose and the nuclei were distributed in a more disorderly manner. After 8 weeks of exercise training, the structure of myofilament was obviously improved (Figure 1D). By electron microscopy, the I-band, the H-band and the M-line were clearly observed in the salinetreated group. There was no breakage of the $\mathrm{Z}$ lines. After treatment with trehalose, the overall shape of the skeletal muscle had been improved. In contrast to this, chloroquine caused the misalignment of muscle fibers, discontinued $\mathrm{Z}$ lines, and the disappearance of the I band. After exercise training, the internal fine structure was clearer, and the myofilament became neater and stouter than observed in the chloroquine treatment group (Figure 1E). These results indicate that exercise was helpful for maintaining the health of the skeletal muscles.

\section{Exercise restored autophagic activity blocked by chloroquine in skeletal muscle}

To evaluate whether exercise could restore autophagic flux after chloroquine treatment, the autophagy level during exercise was determined. Vastus lateralis muscles were collected for Western blotting analysis. The results showed that the LC3-II levels increased in the exercise training groups compared with the untrained animals, leading to a considerable increase in the ratio of LC3-II/Histone. The LC3-II level increased after treatment with trehalose, however, it did not further increase in the group treated with trehalose and exercise together. After treatment with chloroquine, the level of LC3-II increased because chloroquine is an inhibitor of lysosomal enzymes, and it causes the accumulation of autophagosomes. Compared with the chloroquine group, LC3-II levels in the exercise-chloroquine mice significantly decreased (Figure 2A). P62 is known to be the substrate of autophagy ${ }^{[24,25]}$. Our present results showed that the p62 level was high in skeletal muscle of the NS group, while it became lower in the exercise group, the trehalose group, and the exercise-trehalose group. In the chloroquine group, the expression of p62 markedly increased but it was significantly downregulated by exercise training (Figure 2A).

To further evaluate the activation of autophagy, the formation and morphology of autophagosomes were examined by electron microscopy. Compared with the saline control group, more C-shaped double membrane structures and double membrane structures containing mitochondria were observed in mice following exercise training, trehalose administration and exercise and trehalose combined. Many vacuoles of different sizes were found in the group treated with chloroquine because chloroquine blocks the fusion of autophagosomes and lysosomes. The total number and area of the vacuoles were reduced by nearly 30\% after exercise (Figure 2B). The above results demonstrated that autophagy activity could be activated by exercise, and exercise restored the autophagy flux blocked by chloroquine.

\section{Exercise improved mitochondrial function and reduced the apoptotic cell death of skeletal muscles}

Mitochondria provide energy for the activities required for life; however, free radicals are also generated in this process ${ }^{[26]}$. More than $95 \%$ of free radicals are derived from the mitochondrial respiratory chain. Some enzymes, including glutathione peroxidase (GSH) and superoxide dismutase (SOD), can protect cells against damage by superoxide anion radicals ${ }^{[27]}$. Their activities indirectly reflect the cells ability to scavenge oxygen free radicals. Free radicals in the body can cause lipid peroxidation with the production of MDA, therefore the MDA levels reflect the lipid peroxidation intensity. In this study, the level of SOD was significantly increased after 8 weeks of training. Trehalose treatment increased the SOD levels, while chloroquine resulted in a significant reduction. Exercise did not further increase the SOD levels in the trehalose-treated group, whereas it reversed the chloroquine-induced decline in SOD levels (Figure 3A). Similarly, exercise and trehalose also increased GSH levels, and exercise reversed the reduction of GSH levels caused by chloroquine treatment (Figure 3B). We also found that higher levels of MDA were produced in the mice treated with chloroquine, whereas the MDA levels decreased in the trehalose-treated groups. Similarly, exercise inhibited chloroquine-induced elevation of the MDA levels (Figure 3C). We evaluated the morphology of mitochondria to address whether the mitochondria were affected by exercise, trehalose and chloroquine. Electron microscopy revealed that most of the mitochondria in the saline group were normal 
A

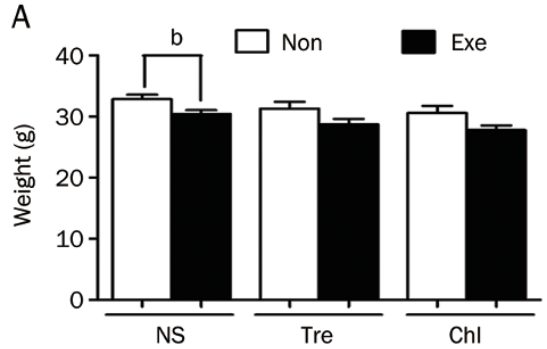

$\mathrm{D}$
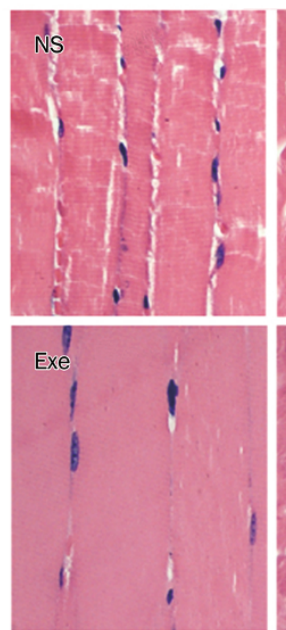

B

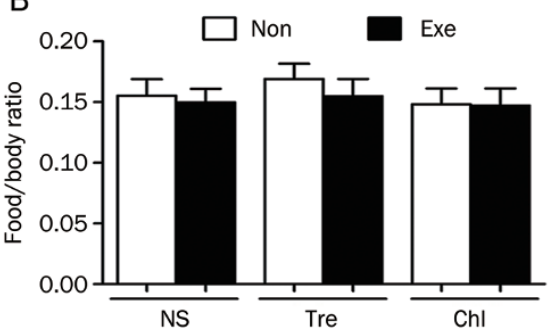

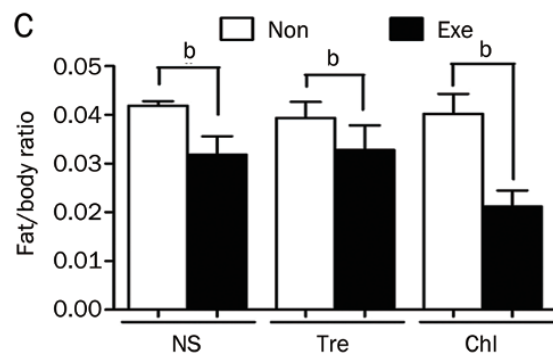

$\mathrm{E}$

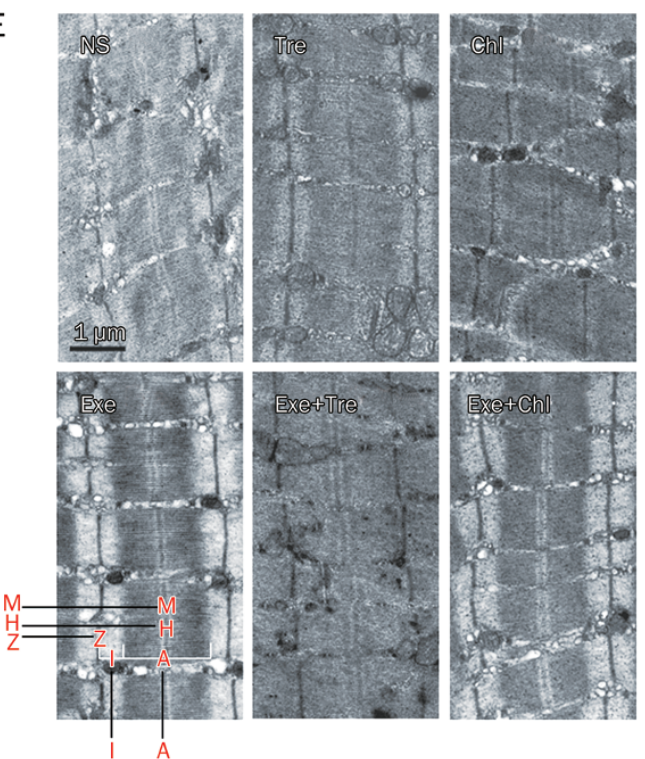

Figure 1. Exercise enhances muscle fibers damaged by chloroquine. All mice were assigned into six groups ( $n=8)$ : untrained mice treated with saline (NS), untrained mice treated with trehalose (Tre), untrained mice treated with chloroquine (Chl), trained mice (Exe), trained mice treated with trehalose (Exe+Tre), and trained mice treated with chloroquine (Exe+Chl). Autophagy stimulator trehalose (1\%) and autophagy inhibitor chloroquine (10 mg/kg) were the positive (+) and negative (-) controls, respectively. (A) The body weight. (B) The food consumption. (C) The fat/body ratio. Exercise decreased the body fat weight. (D) Exercise and autophagy inducer improved the morphology of muscle fibers. Muscle fibers were stained by H\&E staining to expose the morphology, $n=8$. (E) Exercise and autophagy inducer improved the muscle fibers ultra-structure. The internal structure of the skeletal muscle fiber was observed under the lens of the electron microscope from 3 mice in each group. Scale bars: $1 \mu \mathrm{m}$. I: light band, A: dark band, M: M-line, $\mathrm{H}$ : $\mathrm{H}$-band, Z: Z-line. Bar represents mean $\pm \mathrm{SD}, n=8 .{ }^{b} P<0.05,{ }^{\mathrm{c}} P<0.01$ compared with the NS group or the trained group.

with a dark matrix and regular distribution of cristae. Only a few mitochondria showed vacuolization with an enlarged, swollen and pale or even white matrix with few remaining cristae. There were more oval or circular mitochondria and less mitochondrial vacuolization in the trehalose group, while extensive mitochondrial vacuolization was observed in the chloroquine-treated group. Exercise training decreased the mitochondria vacuolization caused by chloroquine treatment (Figure 3D).

When cells commit to apoptosis, genomic DNA breaks into 180-200-bp fragments ${ }^{[28]}$. Exposed $3^{\prime}-\mathrm{OH}$ groups combine with fluorescein-dUTP due to the activity of terminal deoxynucleotidyl transferase (TdT), which can be detected by fluorescence microscopy. Our results showed that there were a few apoptotic cells in the normal control group. There were almost no apoptotic cells in the trehalose group, but there were numerous apoptotic cells in the chloroquine-treated group. However, the number of apoptotic cells in the chloroquine group significantly decreased after exercise (Figure 3E). The number of apoptotic cells was analyzed in all groups (Figure 3F).

Next, we examined the protein levels of Bcl-2 and the Bcl-2/ adenovirus E1B 19-kDa protein-interacting protein 3 (Bnip3) in each group. Western blotting analysis revealed that exercise and trehalose upregulated Bcl-2 expression. Moreover, exercise-induced Bcl-2 expression was decreased by chloroquine treatment (Figure 3G). Bnip3 is a pro-apoptotic protein. Compared with the untrained group, Bnip3 expression was downregulated both in the exercise training group and the trehalose group. The Bnip3 level was greatly increased in the chloroquine group, and it decreased after exercise (Figure $3 \mathrm{H}$ ). The release of cytochrome $c$ (Cyt $c$ ) from the mitochondria induces caspase activation and promotes vital processes such as differentiation and proliferation ${ }^{[29,30]}$. To detect the distribution of Cyt $c$ in the mitochondria and the cytoplasm, we separated the mitochondria from the gastrocnemius. Western blotting analysis revealed that both exercise and trehalose caused 
A
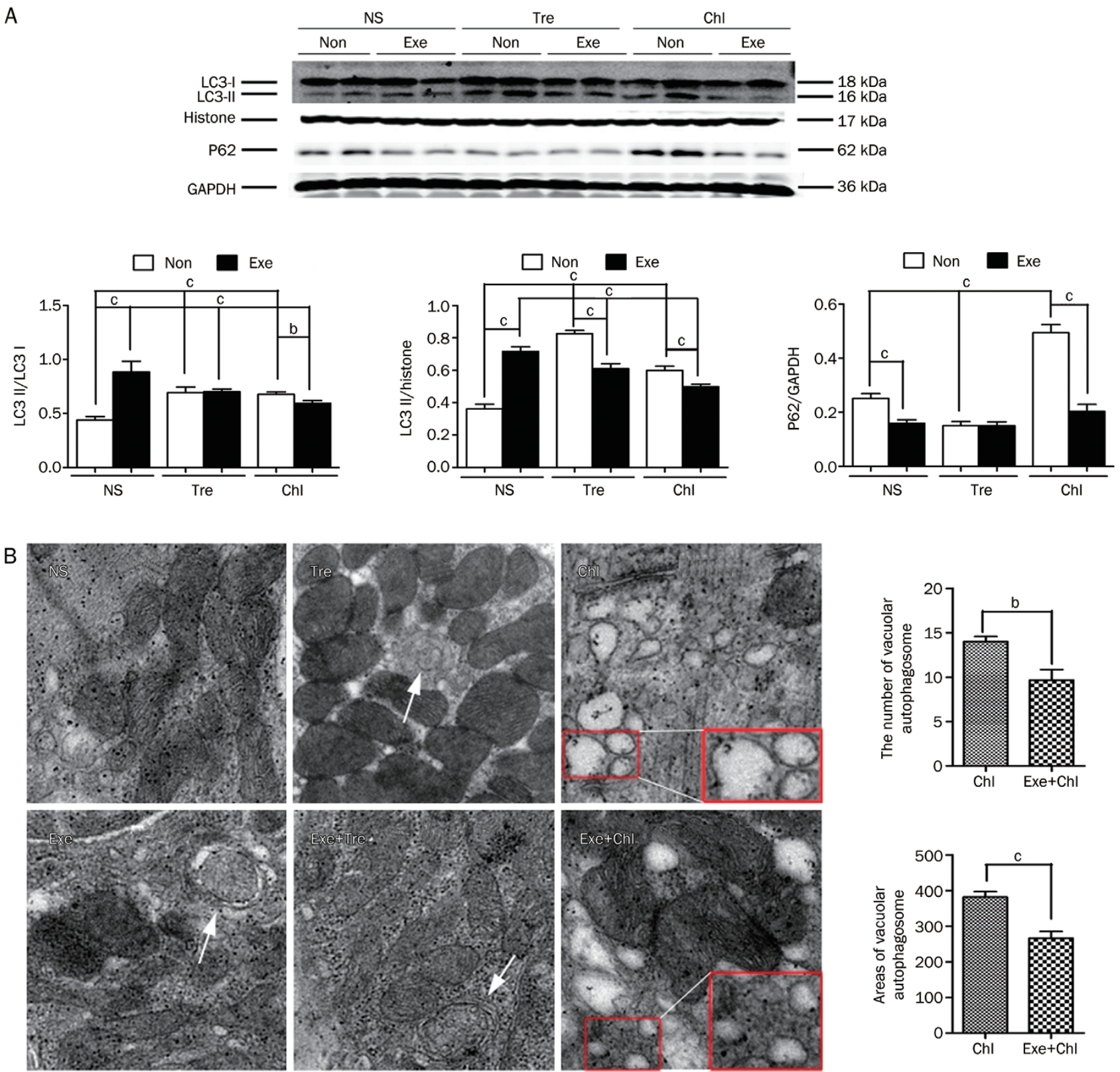

Figure 2. Exercise enhances autophagy activity in skeletal muscle and recovers autophagy level blocked by chloroquine (Chl) in lateralis muscle. (A) Protein expression and quantitative analysis of LC3 and P62. (B) The formation of autophagosomes were induced by exercise (Exe) and trehalose (Tre). White arrows indicate autophagosomes. Autophagosomes in the pictures were enlarged in order to observe the morphology clearly. For Western blotting, levels of GAPDH and Histone protein were used as the loading control. The data were from the same PVDF membrane, and were representative of at least three independent experiments. Quantitative analysis was performed with Sigma Scan Pro 5. Bar represents mean \pm SD from 6 mice in every group. ${ }^{b} P<0.05,{ }^{c} P<0.01$ compared with the NS group or the trained group.

higher Cyt $c$ levels in the mitochondria and lower levels in the cytoplasm, while chloroquine caused lower Cyt $c$ levels in the mitochondria and higher levels in the cytoplasm. Exercise attenuated the chloroquine-induced increase in Cyt $c$ levels in the cytoplasm. The ratio of Cyt $c$ in the mitochondria and cytoplasm showed that the release of Cyt $c$ from the mitochondria was prevented after exercise (Figure 3I).

\section{Discussion}

Autophagy is an important physiological process. Proper regulation of autophagic flux is essential for the homeostasis of skeletal muscles under physiological conditions, particularly in response to stress. In our study, female mice were used because they have been shown to be able to run longer than male mice ${ }^{[31]}$. We generated an optimized exercise model. The 

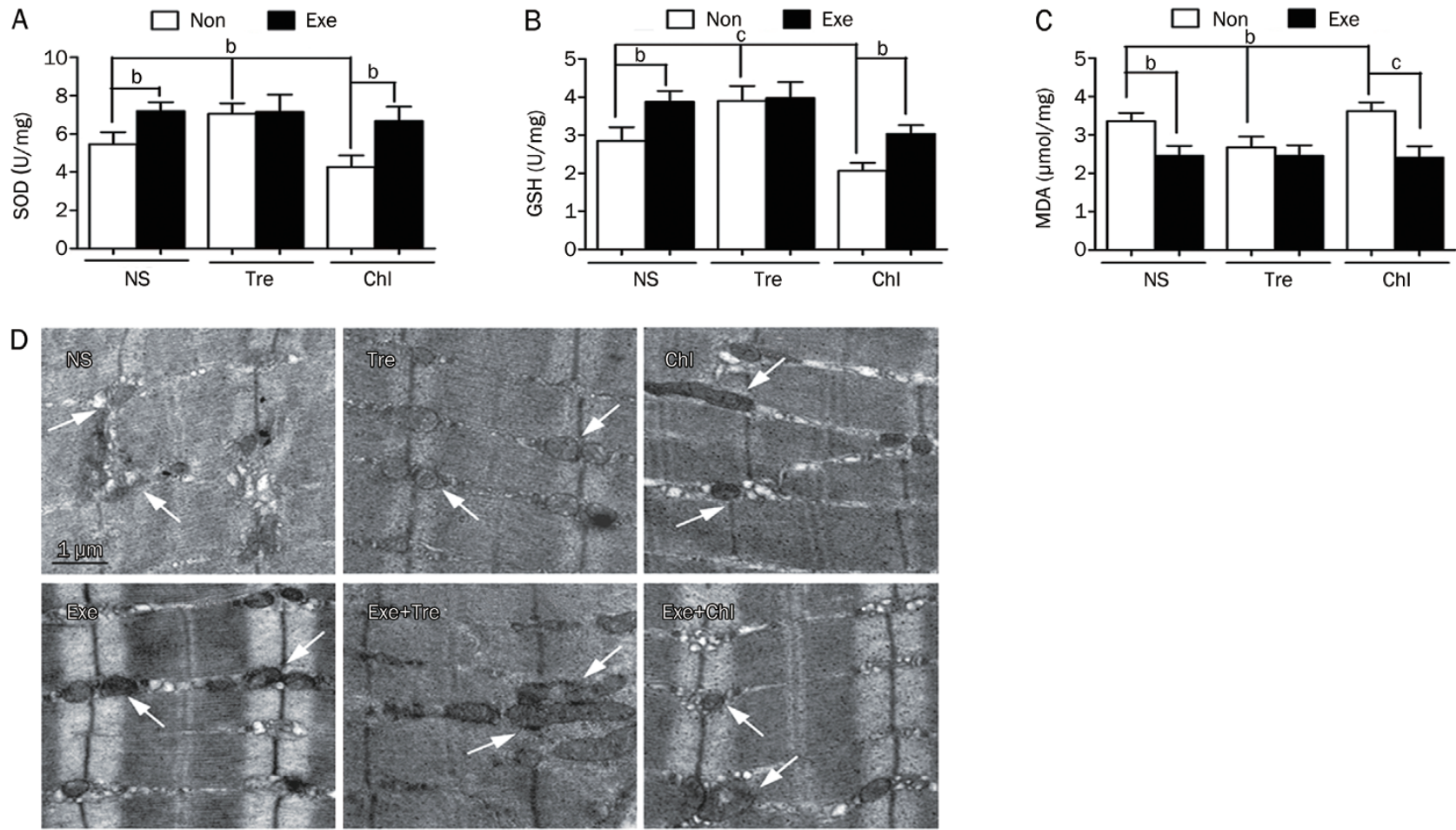

$\mathrm{E}$
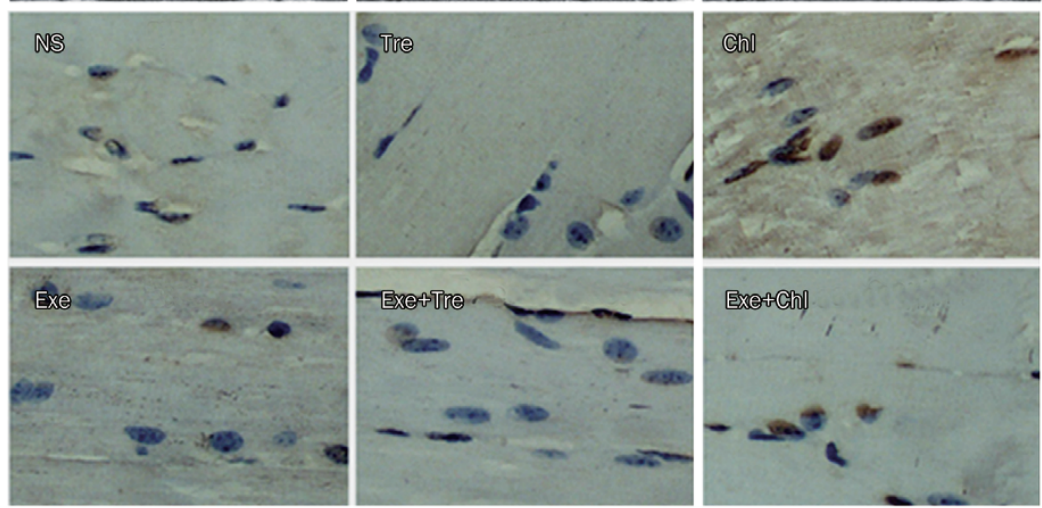

$\mathrm{F}$

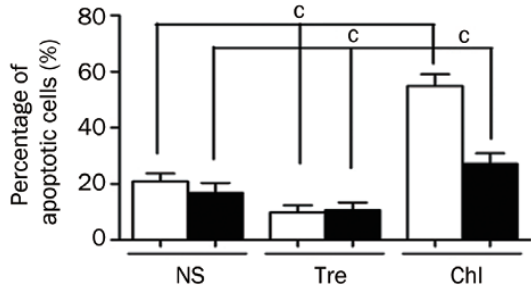

G

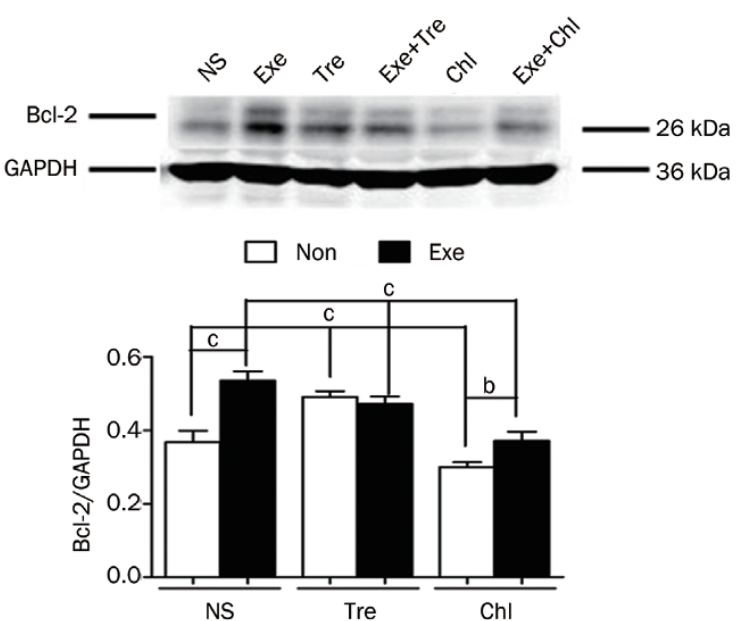

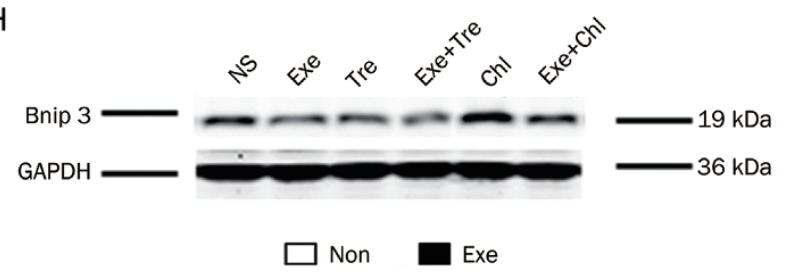

Figure 3A-3H. Exercise enhances the mitochondria function and recovers the toxic effect of chloroquine on skeletal muscles. Quantitative analysis of (A) SOD levels. (B) GSH levels. (C) MDA levels. (D) The morphology of mitochondria. Scale bar=1 $\mu \mathrm{m}$. White arrows indicate mitochondria. (E) The muscle tissue was stained by TUNEL (TdT-mediated dUTP nick end labeling), $n=8$. (F) Quantitative analysis of the number of apoptosis cells. (G) Protein levels and quantitative analysis of Bcl-2. $(\mathrm{H})$ Immunoblotting and quantitative analysis of Bnip 3. Bar represents mean \pm SD from 6 mice in every group. ${ }^{\mathrm{b}} P<0.05,{ }^{\mathrm{c}} P<0.01$ compared with the NS group or the trained group. 

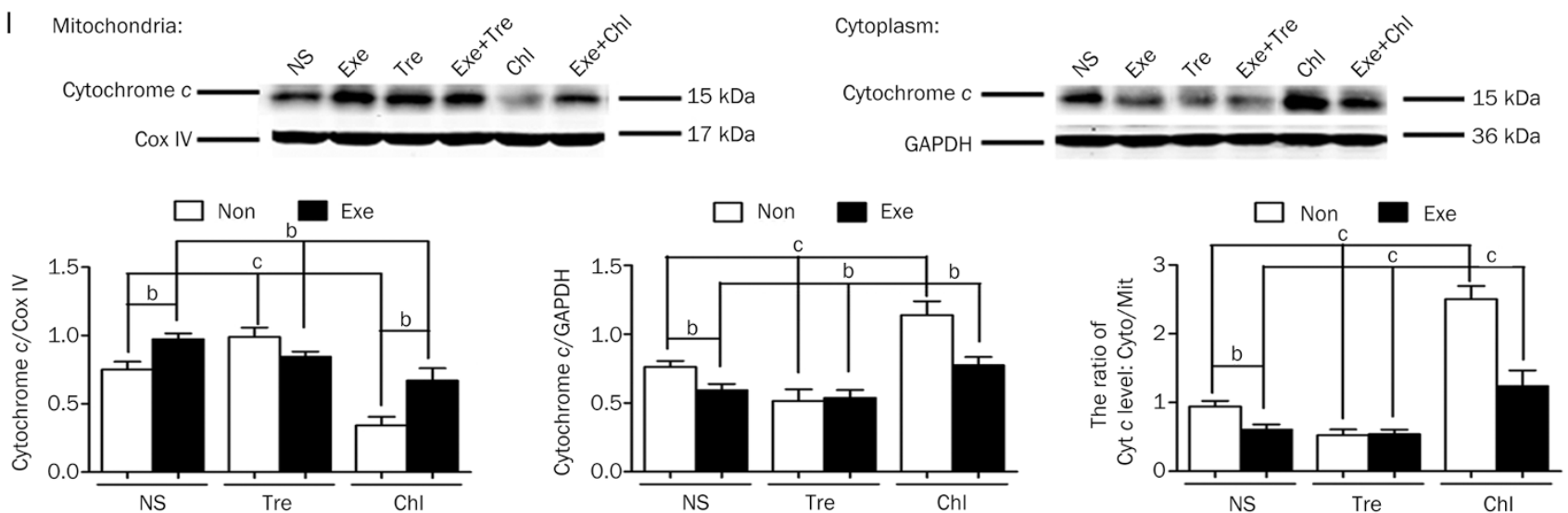

Figure 3I. (I) The level of cytochrome $c$ in mitochondria and cytoplasm. Levels of GAPDH and Cox IV protein were used as the loading control. Quantitative analysis was performed with Sigma Scan Pro 5. Bar represents mean \pm SD from 6 mice in every group. ${ }^{b} P<0.05,{ }^{\circ} P<0.01$ compared with the NS group or the trained group.

intensity of this mode was equivalent to $20 \%$ of the maximum fatigue strength because the longest endurance exercise of mice was approximately $150 \mathrm{~min} /$ times in our study. After 8 weeks of regular exercise, there were more compact and thicker skeletal muscle fibers and a reduction of disordered fibers in the mouse skeletal muscle. In the present study, the activation of autophagy after exercise was confirmed by the induction of LC3-II and the degradation of p62. Additionally, autophagosomes, C-shaped double-membrane structures and engulfment of cytoplasmic materials by autophagosomes were also observed after exercise training.

In this study, the autophagy inducer trehalose $(1 \%)$ and the inhibitor chloroquine $(10 \mathrm{mg} / \mathrm{kg})$ were identified as a positive control and negative control to investigate the role of autophagy in muscle establishment. Trehalose has the potential for application in both the prevention and treatment of neurodegenerative diseases. As a disaccharide, trehalose is a safer autophagy inducer than rapamycin. Trehalose has been approved for use in the United States because it has a protective effect in promoting brain health ${ }^{[32,33]}$. We found that trehalose had similar effects to those induced by exercise. However, the combination of exercise and trehalose did not further enhance autophagy activation, suggesting that a ceiling effect of autophagy activation under physiological conditions may exist.

As an antimalarial drug, one of the side effects of chloroquine is the impairment of muscles. In the present experiment, we found that exercise could ameliorate the detrimental effect of chloroquine on skeletal muscle. Exercise could partially overcome the blockade of autophagy flux caused by chloroquine treatment in the skeletal muscle of mice; this result suggested that exercise might enhance lysosomal function. Salminen et al ${ }^{[34]}$ suggested that strenuous physical exercise induced the necrosis of skeletal muscle fibers, but it increased lysosomal enzyme activities in the surviving muscle fibers. In further research, the effect of exercise on lysosomal synthesis and function should be studied.
Our study also demonstrated that the effects of autophagy induced by exercise on muscle establishment had a role in maintaining the function of mitochondria and inhibiting apoptosis. Numerous mitochondria with normal morphology were observed in the skeletal muscle of exercise-trained mice. The ability to remove free radicals had been significantly improved after exercise. The trained mice had less apoptotic muscle cells. Expression of the anti-apoptotic protein Bcl-2 was significantly increased, and the mitochondrial apoptotic protein Bnip 3 was downregulated. Exercise also prevented Cyt $c$ release from mitochondria into the cytoplasm. Similar to exercise, trehalose greatly enhanced mitochondrial function.

In summary, our results showed that exercise and trehalose increased the autophagy levels under normal physiological condition, and they ameliorated the detrimental effects of chloroquine on skeletal muscles. The beneficial effects of exercise on skeletal muscle fitness and chloroquine toxicity are associated with autophagy activation.

\section{Acknowledgements}

We are grateful to Dr Fiona M MENZIES for her careful revision and critical comments on this paper. This work were supported by grants from the Priority Academic Program Development of Jiangsu Higher Education Institutions (PADA) and Jiangsu Key Laboratory of Translational Research and Therapy for Neuro-Psycho-Diseases and College of Pharmaceutical Sciences, Soochow University, China (BM2013003).

\section{Author contribution}

Dan JIANG and Xuan LU carried out the experimental studies; Hong-jian GAO carried out the electron microscopy; Kai CHEN wrote and revised the manuscript; Fang LIN and Zheng-hong QIN designed the research and reviewed the manuscript.

\section{References}

1 Warburton DE, Nicol CW, Bredin SS. Health benefits of physical 
activity: the evidence. CMAJ 2006; 174: 801-9.

2 Mizushima N, Levine B, Cuervo AM, Klionsky DJ. Autophagy fights disease through cellular self-digestion. Nature 2008; 451: 1069-75.

3 Sandri M. Autophagy in skeletal muscle. FEBS Lett 2010; 584: 1411-6.

4 Masiero E, Agatea L, Mammucari C, Blaauw B, Loro E, Komatsu M, et al. Autophagy is required to maintain muscle mass. Cell Metabolism 2009; 10: 507-15.

5 Fukuda T, Ahearn M, Roberts A, Mattaliano RJ, Zaal K, Ralston E, et al. Autophagy and mistargeting of therapeutic enzyme in skeletal muscle in Pompe disease. Mol Ther 2006; 14: 831-9.

6 Arndt V, Dick N, Tawo R, Dreiseidler M, Wenzel D, Hesse M, et al. Chaperone-assisted selective autophagy is essential for muscle maintenance. Curr Biol 2010; 20: 143-8.

7 Masiero E, Agatea L, Mammucari C, Blaauw B, Loro E, Komatsu M, et al. Autophagy is required to maintain muscle mass. Cell Metab 2009; 10: 507-15.

8 Sung YH, Kim SC, Hong HP, Park CY, Shin MS, Kim CJ, et al. Treadmill exercise ameliorates dopaminergic neuronal loss through suppressing microglial activation in Parkinson's disease mice. Life Sci 2012; 91: 1309-16.

9 Schenkman M, Hall DA, Baron AE, Schwartz RS, Mettler P, Kohrt WM. Exercise for people in early- or mid-stage Parkinson disease: a 16-month randomized controlled trial. Phys Ther 2012; 92: 1395410.

10 Ogura Y, lemitsu M, Naito H, Kakigi R, Kakehashi C, Maeda S, et al. Single bout of running exercise changes LC3-II expression in rat cardiac muscle. Biochem Biophys Res Commun 2011; 414: 756-60.

$11 \mathrm{Kim}$ YA, Kim YS, Song W. Autophagic response to a single bout of moderate exercise in murine skeletal muscle. J Physiol Biochem 2011; 68: 229-35.

12 Jamart C, Benoit N, Raymackers JM, Kim HJ, Kim CK, Francaux M. Autophagy-related and autophagy-regulatory genes are induced in human muscle after ultraendurance exercise. Eur J Appl Physiol 2012; 112: 3173-7.

13 Wohlgemuth SE, Lees HA, Marzetti E, Manini TM, Aranda JM, Daniels $\mathrm{MJ}$, et al. An exploratory analysis of the effects of a weight loss plus exercise program on cellular quality control mechanisms in older overweight women. Rejuvenation Res 2011; 14: 315-24.

14 He C, Bassik MC, Moresi V, Sun K, Wei Y, Zou Z, et al. Exerciseinduced BCL2-regulated autophagy is required for muscle glucose homeostasis. Nature 2012; 481: 511-5.

15 Irrcher I, Adhihetty PJ, Joseph AM, Ljubicic V, Hood DA. Regulation of mitochondrial biogenesis in muscle by endurance exercise. Sports Med 2003; 33: 783-93.

16 Wu JJ, Quijano C, Chen E, Liu H, Cao L, Fergusson MM, et al. Mitochondrial dysfunction and oxidative stress mediate the physiological impairment induced by the disruption of autophagy. Aging (Albany NY) 2009; 1: 425-37.

17 Dobrowolny G, Aucello M, Rizzuto E, Beccafico S, Mammucari C, Boncompagni $\mathrm{S}$, et al. Skeletal muscle is a primary target of SOD1G93Amediated toxicity. Cell Metab 2008; 8: 425-36.

18 Pietri-Rouxel F, Gentil C, Vassilopoulos S, Baas D, Mouisel E, Ferry A, et al. DHPR alpha1S subunit controls skeletal muscle mass and morphogenesis. EMBO J 2010; 29: 643-54.

19 Tolkovsky AM. Autophagy thwarts muscle disease. Nat Med 2010; 16: 1188-90.

20 Sarkar S, Davies JE, Huang Z, Tunnacliffe A, Rubinsztein DC. Trehalose, a novel mTOR-independent autophagy enhancer, accelerates the clearance of mutant huntingtin and alpha-synuclein. J Biol Chem 2007; 282: 5641-52.

21 Yang YP, Hu LF, Zheng HF, Mao CJ, Hu WD, Xiong KP, et al. Application and interpretation of current autophagy inhibitors and activators. Acta Pharmacol Sin 2013; 34: 625-35.

22 Suzuki T, Nakagawa M, Yoshikawa A, Sasagawa N, Yoshimori T, Ohsumi Y, et al. The first molecular evidence that autophagy relates rimmed vacuole formation in chloroquine myopathy. J Biochem 2002; 131: 647-51.

23 Ramos L, Wetzels AM. Low rates of DNA fragmentation in selected motile human spermatozoa assessed by the TUNEL assay. Hum Reprod 2001; 16: 1703-7.

24 Komatsu M, Waguri S, Chiba T, Murata S, Iwata J, Tanida I, et al. Loss of autophagy in the central nervous system causes neurodegeneration in mice. Nature 2006; 441: 880-4.

25 Raben N, Hill V, Shea L, Takikita S, Baum R, Mizushima N, et al. Suppression of autophagy in skeletal muscle uncovers the accumulation of ubiquitinated proteins and their potential role in muscle damage in Pompe disease. Hum Mol Genet 2008; 17: 3897-908.

26 Criscuolo F, Gonzalez-Barroso Mdel M, Le Maho Y, Ricquier D, Bouillaud F. Avian uncoupling protein expressed in yeast mitochondria prevents endogenous free radical damage. Proc Biol Sci 2005; 272 : 803-10.

27 Alper G, Girgin FK, Ozgonul M, Mentes G, Ersoz B. MAO inhibitors and oxidant stress in aging brain tissue. Eur Neuropsychopharmacol 1999; 9: 247-52.

28 Elmore S. Apoptosis: a review of programmed cell death. Toxicol Pathol 2007; 35: 495-516.

29 Garrido C, Galluzzi L, Brunet M, Puig PE, Didelot C, Kroemer G. Mechanisms of cytochrome $c$ release from mitochondria. Cell Death Differ 2006; 13: 1423-33.

30 Arnoult D, Grodet A, Lee YJ, Estaquier J, Blackstone C. Release of OPA1 during apoptosis participates in the rapid and complete release of cytochrome $c$ and subsequent mitochondrial fragmentation. J Biol Chem 2005; 280: 35742-50.

31 Girard I, McAleer MW, Rhodes JS, Garland T Jr. Selection for high voluntary wheel-running increases speed and intermittency in house mice (Mus domesticus). J Exp Biol 2001; 204: 4311-20.

32 Colaco C, Kampinga J, Roser B. Amorphous stability and trehalose. Science 1995; 268: 788.

33 Tanaka M, Machida Y, Niu S, Ikeda T, Jana NR, Doi H, et al. Trehalose alleviates polyglutamine-mediated pathology in a mouse model of Huntington disease. Nat Med 2004; 10: 148-54.

34 Salminen A, Vihko V. Autophagic response to strenuous exercise in mouse skeletal muscle fibers. Virchows Arch B Cell Pathol Incl Mol Pathol 1984; 45: 97-106. 